\title{
Ovarian tumor diagnosed during pregnancy \& their management
}

\author{
Harsha Khullar*, Geeta Mediratta and Sharmistha Garg \\ Institute of Obstetrics \& Gynecology, Sir Ganga Ram Hospital, New Delhi, India
}

\begin{abstract}
The incidence of ovarian tumor in pregnancy is quite rare approximating to 1 in 190 pregnancies. Scanty evidence is available for the treatment of this condition due to lack of RCTs and cohort studies. However, only 1-6\% of ovarian tumors are reported to be malignant in nature during pregnancy. We report 6 cases of ovarian malignancies which depicts the difficulty of making this rare diagnosis along with their brief clinical presentation, diagnosis and therapeutic approach. The findings from these cases describe the interventions which ameliorates patients' quality of life.
\end{abstract}

\section{Key points}

- Ovarian tumor is the fifth most common cancer

- Only 3 to $5 \%$ of ovarian tumors in pregnancy are malignant.

- Signs and symptoms include incidental finding, abdominal or back pain.

- High levels of Maternal Serum AFP, Inhibin A obtained during aneuploidy screening can be a sign of GCT

- Adequate surgical staging is important for stage I cancer.

- Ideal time for surgery is in second trimester of pregnancy

- Treatment in each case should be individualized

- Chemotherapy can be given antenatally but with prior consents.

\section{Case series}

A 30-year-old female came at 10 weeks of pregnancy with an 8 $\mathrm{cm}$ cyst in left adnexa. On investigation, Ultrasound showed features of a dermoid cyst. Patient was advised to have laparoscopy/ ovarian cystectomy in the second trimester which she refused. However, pregnancy progressed well, and she had to undergo caesarean section at term in view of breech presentation. On CS the cyst was also enucleated and healthy ovarian tissue was preserved. Cut section showed sebaceous material, teeth, bone and hair. On histopathological examination, mature cystic teratoma was reported (Figure 1).

A young multigravida had normal vaginal delivery at term. She was diagnosed to have $10 \mathrm{~cm}$ ovarian cyst during antenatal period. $6 \mathrm{hrs}$ after delivery patient complained of severe pain abdomen. Uterus was well contracted. Emergency ultrasound done showed haemorrhage in cyst (Figure 2).

A-30-year-old young primigravida presented with early pregnancy with solid ovarian tumor on one side. Serial ultrasound did not show any increase in the size of tumour. Laparoscopy done and patient underwent one side salpingo-oopherectomy. Other side tube and ovary were normal. Histopathology report suggested serous cyst adenoma with borderline malignancy (Figure 3).

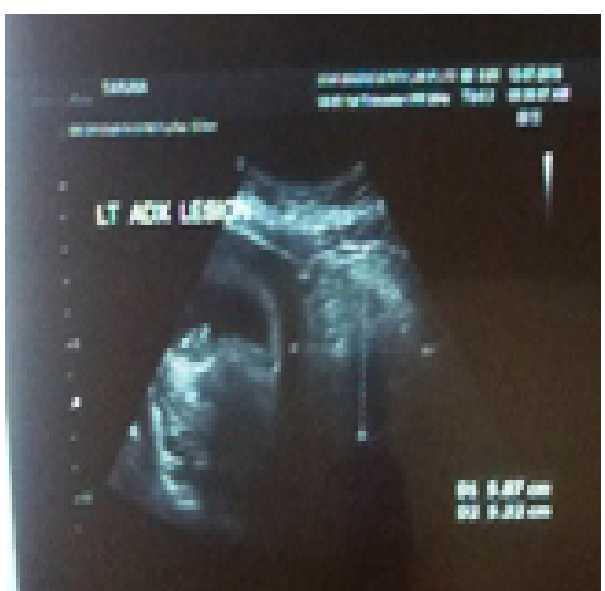

Figure 1. USG depicting mature cystic teratoma in left adnexa

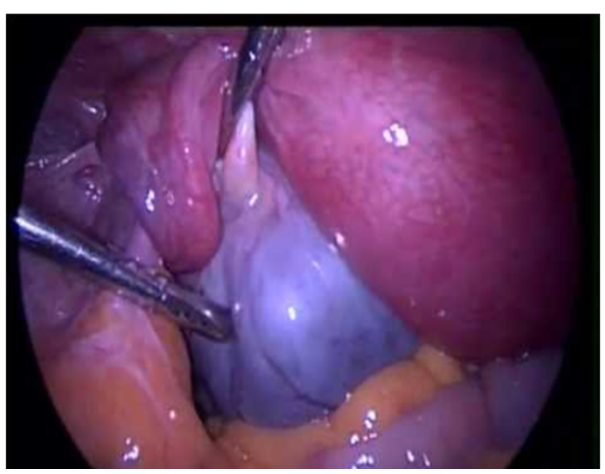

Figure 2. Hemorrhagic cyst

${ }^{\star}$ Correspondence to: Harsha Khullar, Vice-Chairperson\& Senior Consultant, Institute of Obstetrics \& Gynecology, Sir Ganga Ram Hospital, New Delhi, India, E-mail: harsha_khullar@yahoo.com

Received: May 25, 2020; Accepted: June 16, 2020; Published: June 19, 2020 


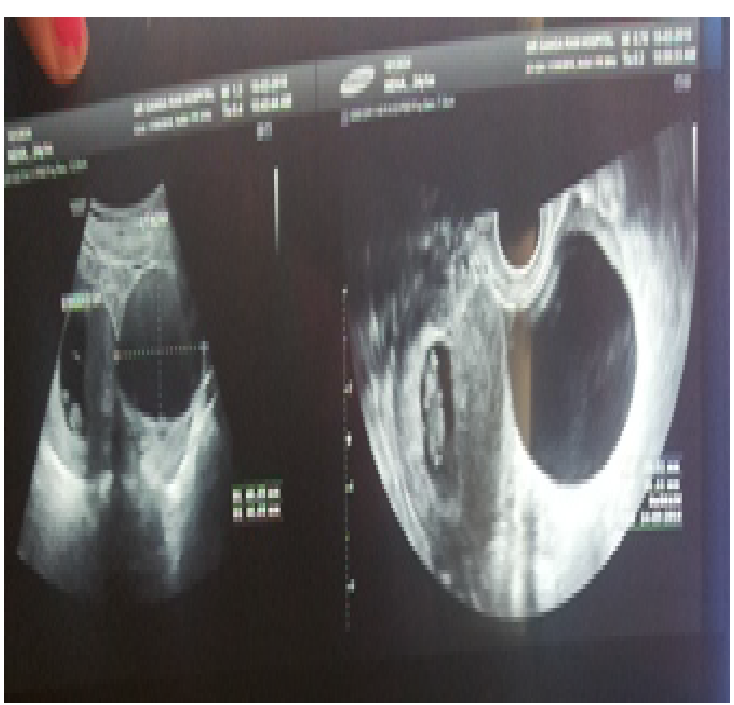

Figure 3. Serous Cyst Adenoma

Elderly primigravida with IVF pregnancy presented with cyst in both ovaries. She was followed up and by $16 \mathrm{wks}$ pregnancy, the cysts had disappeared.

Treated case of endometriosis with infertility underwent LSCS at term. Both ovaries had large cysts. Cystectomy was done. Histopathology report suggested endometriosis.

Young patient with previous history of ovarian cystectomy (simple cyst) presented at 14 weeks pregnancy with ovarian cyst of size $8-10 \mathrm{~cm}$. It was increasing in size, so she underwent laparoscopy with removal of intact ovarian cyst at 20 weeks pregnancy. Histopathology report suggested serous cyst adenocarcinoma. She was followed up and at 34 weeks underwent LSCS with staging laparotomy. She wanted her uterus to be preserved. Omentum lymph node and other ovary were found negative for malignancy. For last 7 years, she is under follow up and is doing well.

\section{Discussion}

Ovarian Dermoid cysts $<6 \mathrm{~cm}$ is not expected to grow during pregnancy or to cause complications in pregnancy and labor. 50 percent of Epithelial Ovarian Cancer (EOC) is detected during pregnancy (Low Malignant Potential) and rest $50 \%$ is invasive in character. Germ Cell Tumor comprises of $75 \%$ dysgerminoma, $25 \%$ EST, immature teratoma and mixed. Most of the GCT is unilateral. $10-15 \%$ dysgerminoma are bilateral. Sex cords tumors include $50 \%$ granulosa cell tumor, 35\% sertoli leydig cell tumor and rest $15 \%$ are unclassified or stromal. Asymptomatic masses $>10 \mathrm{~cm}$ present after $1^{\text {st }}$ trimester or tumors which are solid or having solid and cystic areas/ papillary are usually malignant. Resection is the line of treatment in case of a malignant malignancy. For Benign looking tumors, expectant management is preferred [1]. Diagnosis can be made by appropriate history recording, sonological features, resecting the ovarian neoplasm and Biopsy. The Clinical features of neoplasm include Asymptomatic/ nonspecific symptoms, V/E in early pregnancy, acute pain, and unexplained elevated markers during antenatal screening. Markers like CA 125 are unlikely to be markedly elevated solely because of pregnancy. Range between 1000-10,000 likely but not always related to cancer and 75-150 could be either pregnancy related. AFP normally rises during pregnancy. AFP levels associated with ovarian cancer can be much higher MOMs. LDH is not increased in normal pregnancy but increases in patients with HELLP, Pre-eclampsia and in women with dysgerminoma. HCG cannot be used due to its physiologic increase in pregnancy. Inhibin A is made in developing placenta. All antigens studied, except CA 153, are oncofetal. The presence of considerable concentrations of AFP, hCG, CEA, CA125, SCC, MCA, TPS, CA 199, and PSA in AF during pregnancy may be attributed to their involvement in biological functions associated with fetal development, differentiation, and maturation. MS CEA, CA 153, and CA 199, in contrast to all the others, are not influenced significantly by pregnancy and thus remain reliable tumor markers in monitoring malignancy in pregnant patients [2]. Familiarity with the clinicopathologic and sonographic features of common and uncommon adnexal masses in pregnancy is important for diagnosis and treatment [3]. Surgical intervention must be done after first trimester and became functioning ovarian cysts resolve; drug induced teratogenesis is reduced, NT scan rules out aneuploidy, Corpus Luteum function has been replaced by placenta by this period of gestation Rise in the frequency of adnexal masses exposed during pregnancy concurrently occurs with the prenatal ultrasound adoption. However, majority of such masses may resolve by second trimester. Unrelenting masses poses a great risk for episodes like torsion, rupture and obstruction of labour. These events may lead to emergency surgical intervention with elevated risk for unfavourable outcome for mother and the fetus. Additionally, a small risk of cancer still prevails and therefore, prolong delay in the diagnosis must be avoided. Surgical resection of constant adnexal mass should be done at approximately 16 to 20 weeks of gestation. In $5 \%$ malignant cases of adnexal masses, accurate staging must be done. However, in certain cases chemotherapy can be initiated [4]

If a malignancy is suspected, surgical intervention via midline vertical incision is the choice. Laparoscopy is recommended in the case of Low Malignancy Potential. In case of a malignancy, surgical staging assess other ovary. If pregnancy is desired, hysterectomy should not be performed. If the diagnosed malignancy is ovarian cancer, then cytoreduction is attempted. Choice of treatment for women diagnosed with advanced stage ovarian cancer before delivery is radical surgery. Avoid lymph node dissection in case of Germ cell tumor such as Endodermal sinus tumor. Widespread usage of ultrasonography in early pregnancy phase has helped the identification of incidental adnexal masses more frequent. Although, high resolution ultrasound has made observation, a practical option. Surgical treatment is warranted at risk of clinically symptomatic, torsion, or rupture masses suspected for malignancy. In well-equipped tertiary care centers, laparoscopy has depicted success in pregnancy and is safe in experienced hands and equipped armamentarium [5]. FIGO stage III endometrioid ovarian carcinoma diagnosed during 22 weeks of pregnancy was treated with chemotherapy while preserving the pregnancy [6]. During pregnancy, chemotherapy must be considered in second trimester if a diagnosis of epithelial ovarian cancer is made. However, cancer of ovary during pregnancy is uncommon. Majority of chemotherapy sessions are administered in combination with cisplatinum. However, scanty evidence is available regarding the use of Paclitaxel with carboplatin during pregnancy and no adverse episode has been reported in the fetus [7]. Since scanty evidence is available regarding the best possible therapeutic approach to epithelial ovarian cancer during pregnancy. Individual approach must be addressed for different cases. In this rare and challenging situation, treatment in tertiary care centre must be intended $[8,9]$. Adnexal mass during caesarean delivery poses a positive favourable prognosis in terms of maternal and fetal outcomes [10]. Serous neoplasm of low malignant potential depicts aggressive microscopic and clinical features during pregnancy. However, the 
features appear to revert back in case of pregnancy termination [11]. After $7^{\text {th }}$ week of gestation, ovarian tumors $>10 \mathrm{~cm}$ diameter or having a teratoma component can be resected to avoid complications. This indirectly reduces the risk for malignancy and emergency surgery. No concrete evidence is suggestive of fetal loss risk during $7^{\text {th }}$ week of gestation [12]. Conservative Management is the line of management employed for the optimal management of ovarian tumors in pregnancy [13].

\section{Conclusion}

Ovarian tumor is the fifth most common cancer diagnosed during pregnancy after breast, thyroid, cervical and Hodgkin's disease. This tumor can be categorized into two types as Benign and Malignant. Examples of benign entities include simple cyst, theca luteal cyst and luteoma. 1-6\% adnexal masses detected during pregnancy are malignant. Only 3 to $5 \%$ of ovarian tumors in pregnancy are malignant. However, majority of the complicating tumors in pregnancy are either borderline or malignant in nature. Signs and symptoms include incidental finding, abdominal or back pain. High levels of Maternal Serum AFP, Inhibin A obtained during aneuploidy screening can be a sign of GCT. Surgical resection is suggested rather than expectant management of asymptomatic masses present after first trimester that are $>10 \mathrm{~cm}$ or have solid/ papillary area. Adequate surgical staging is important for stage I cancer. Ideal time for surgery is in second trimester of pregnancy Treatment in each case should be individualized. Chemotherapy can be given antenatally but with prior consents. Prognosis in patients detected with ovarian tumors in pregnancy is good.

\section{References}

1. Caspi B, Levi R, Appelman Z, Rabinerson D, Goldman G, et al. (2000) Conservative management of ovarian cystic teratoma during pregnancy and labor. Am J Obstet Gynecol 182: 503-505. [Crossref]
2. Sarandakou A, Protonotariou E, Rizos D (2007) Tumor markers in biolog ical fluids associated with pregnancy. Crit Rev Clin Lab Sci 44: 151-178. [Crossref]

3. Chiang G, Levine D (2004) Imaging of adnexal masses in pregnancy. J Ultrasound Med 23: 805-819. [Crossref]

4. Giuntoli RL 2nd, Vang RS, Bristow RE (2006).Evaluation and management of adnexal masses during pregnancy. Clin Obstet Gynecol 49: 492-505. [Crossref]

5. Yakasai IA, Bappa LA (2012) Diagnosis and management of adnexal masses in pregnancy. J Surg Tech Case Rep 4: 79-85. [Crossref]

6. Picone O, Lhommé C, Tournaire M, Pautier P, Camatte S, et al. (2004) Preservation of pregnancy in a patient with a stage IIIB ovarian epithelial carcinoma diagnosed at 22 weeks of gestation and treated with initial chemotherapy: case report and literature review. Gynecol Oncol 94: 600-604. [Crossref]

7. Méndez LE, Mueller A, Salom E, González-Quintero VH (2003) Paclitaxel and carboplatin chemotherapy administered during pregnancy for advanced epithelial ovarian cancer. Obstet Gynecol 102: 1200-1202. [Crossref]

8. Yen CF, Lin SL, Murk W, Wang CJ, Lee CL, et al. (2009) Risk analysis of torsion and malignancy for adnexal masses during pregnancy. Fertil Steril 91: 1895-1902. [Crossref]

9. Grimm D, Woelber L, Trillsch F, Keller-v Amsberg G, Mahner S (2014) Clinica management of epithelial ovarian cancer during pregnancy. Eur J Cancer 50: 963-971. [Crossref]

10. Baser E, Erkilinc S, Esin S, Togrul C, Biberoglu E, et al. (2013) Adnexal masses encountered during cesarean delivery. Int J Gynaecol Obstet 123: 124-126. [Crossref]

11. Mooney J, Silva E, Tornos C, Gershenson D (1997) Unusual features of serous neoplasms of low malignant potential during pregnancy. Gynecol Oncol 65: 30-35. [Crossref]

12. Wang PH, Chao HT, Yuan CC, Lee WL, Chao KC, et al. (1999) Ovarian tumors complicating pregnancy. Emergency and elective surgery. J Reprod Med 44: 279-287. [Crossref]

13. 13.Lee GS, Hur SY, Shin JC, Kim SP, Kim SJ (2004) Elective vs. conservative management of ovarian tumors in pregnancy. Int J Gynaecol Obstet 85: 250-254. [Crossref]

Copyright: (C2020 Khullar H. This is an open-access article distributed under the terms of the Creative Commons Attribution License, which permits unrestricted use, distribution, and reproduction in any medium, provided the original author and source are credited. 